\title{
A NOTE ON CAUSALITY AND INVERTIBILITY OF A GENERAL BILINEAR TIME SERIES MODEL
}

\author{
JIAN LIU, ${ }^{*}$ University of British Columbia
}

In Liu and Brockwell (1988), a sufficient condition for the existence of a strictly stationary, causal and ergodic general bilinear time series (without assuming subdiagonality) has been derived which requires the existence of moments of the noise sequence up to a certain order. However, as demonstrated by Liu (1989), there exists a class of strictly stationary, causal and ergodic bilinear models with noise sequences which may have infinite variance. Also, it is noted that, like other conditions, with the increasing of bilinear orders, the order of the spectral matrix increases rapidly; this may cause some difficulty, depending on the power of the available computing facilities, in the practical checking of stationarity of the bilinear model concerned. The aim of this letter is to present a relatively general but simple condition which will ensure the existence of a strictly stationary, causal and ergodic general bilinear time series model. Consequently, a simple invertibility condition is derived.

Following Granger and Anderson (1978a) and Subba Rao and Gabr (1984), a real-valued time series $\left\{X_{t}\right\}, t \in Z$, is said to be a general bilinear time series if it satisfies the following equations:

$$
X_{t}=\sum_{i=1}^{p} \phi_{i} X_{t-i}+\sum_{j=1}^{q} \theta_{j} Z_{t-j}+Z_{t}+\sum_{i=1}^{m} \sum_{j=1}^{l} b_{i j} X_{t-i} Z_{t-j}, \quad t \in Z,
$$

where $\left\{Z_{t}\right\}$ is an i.i.d. sequence of random variables, $\phi_{i}, \theta_{j}$ and $b_{i j}$ are non-random constants and $p, q, m$ and $l$ are some known non-negative integers. The bilinear time series $\left\{X_{t}\right\}$ is said to be causal if there exists a measurable function $g(\cdot)$ mapping from $R^{\infty}$ to $R^{1}$ such that $X_{t}=g\left(Z_{t}, Z_{t-1}, \cdots\right)$ for all $t \in Z$ and invertible if $Z_{t}=g\left(X_{t}, X_{t-1}, \cdots\right)$ for some measurable function $g(\cdot)$.

I shall assume, without loss of generality, that $p=m$, since otherwise zeroes of $\phi_{i}$ and $b_{i j}$ can be filled in. Define the $p \times p$ companion random matrix $B(t)$ by

$$
B(t):=\left(\begin{array}{ccccc}
\phi_{1}+\sum_{j=1}^{l} b_{1 j} Z_{t-j} & \phi_{2}+\sum_{j=1}^{l} b_{2 j} Z_{t-j} & \cdots & \cdots & \phi_{p}+\sum_{j=1}^{l} b_{p j} Z_{t-j} \\
1 & 0 & \cdots & 0 & 0 \\
\vdots & 0 & & & \vdots \\
0 & 0 & \cdots & 1 & 0
\end{array}\right) .
$$

Then define, for each $p \times p$ matrix $A=\left[a_{i j}\right]$, the norm $\|A\|$ of $A$ by $\|A\|:=\max _{i}\left\{\sum_{j=1}^{p}\left|a_{i j}\right|\right\}$ and the matrix operator $|\cdot|^{\gamma}$, for each $\gamma>0$, by $|A|^{\gamma}:=\left[\left|a_{i j}\right|^{\gamma}\right]$. It is easily seen that for the matrix operator $|\cdot|^{\gamma}$, where $\gamma \in(0,1]$, and sequence of $p \times p$ matrices $A_{n}$,

$$
\left|\sum_{n} A_{n}\right|^{\gamma} \leqq \sum_{n}\left|A_{n}\right|^{\gamma}, \quad\left|\prod_{n} A_{n}\right|^{\gamma} \leqq \prod_{n}\left|A_{n}\right|^{\gamma},
$$

where the matrix relation $A \leqq B$ denotes the elementwise relation $a_{i j} \leqq b_{i j}$ for all $i$ and $j$.

It can be shown that if $E\left\{\ln ^{+}\left[\sum_{i=1}^{p}\left|\phi_{i}+\sum_{j=1}^{l} b_{i j} Z_{t-j}\right|\right]<\infty\right.$,

$$
\delta:=\limsup _{n \rightarrow \infty} n^{-1} E\{\ln \|B(t) B(t-1) \cdots B(t-n)\|\}
$$

Received 3 March 1989; revision received 14 August 1989.

* Postal address: Department of Statistics, 2021 West Mall, University of British Columbia, Vancouver, B.C., Canada V6T 1W5. 
always exists (may be infinite) and furthermore,

$$
\delta:=\limsup _{n \rightarrow \infty} n^{-1} \ln \|B(t) B(t-1) \cdots B(t-n)\| \leqq E\left\{\ln \left\|\prod_{j=1}^{p} B(j)\right\|\right\}, \quad \text { a.s. },
$$

where for each $x \in \boldsymbol{R}^{1}, \ln ^{+} x:=\max \{0, \ln x\}$. Though the condition $\delta<0$ could be used as a sufficient condition for stationarity, it is of little use for the practical checking of stationarity since this condition involves the limit of products of infinitely many random matrices. This leads to the following slightly stronger condition.

Theorem 1. Suppose that $E\left\{\ln ^{+}\left|Z_{1}\right|\right\}<\infty$. If

$$
E\left\{\ln \left\|\prod_{j=1}^{p} B(t-j)\right\|\right\}<0
$$

then there exists a unique strictly stationary, causal and ergodic time series satisfying Equation (1) which can be expressed as the first component of almost sure sum of the infinite series,

$$
\Theta \tilde{Z}(t)+\sum_{n=1}^{\infty}\left[\prod_{j=1}^{n} B(t-j)\right] \Theta \tilde{Z}(t-n)
$$

where the $p \times(1+q)$ matrix $\Theta$ is defined by

$$
\Theta:=\left(\begin{array}{ccccc}
1 & \theta_{1} & \cdots & \theta_{q-1} & \theta_{1} \\
0 & 0 & \cdots & 0 & 0 \\
0 & 0 & \cdots & 0 & 0 \\
\vdots & & & & \vdots \\
0 & 0 & \cdots & 0 & 0
\end{array}\right)
$$

and the random vector $\tilde{Z}(t)$ is defined by $\tilde{Z}(t):=\left(Z_{t}, Z_{t-1}, \cdots, Z_{t-q}\right)^{\prime}$. Also, the uniqueness of solutions of (1) is in fact the uniqueness of solutions in the class of solutions of (1) which are bounded in probability.

Example 1. Consider the bilinear model

$$
X_{t}=\sum_{i=1}^{2} \phi_{i} X_{t-i}+\sum_{i=1}^{2} \sum_{j=1}^{2} b_{i j} X_{t-i} Z_{t-j}+Z_{t} .
$$

In this case, we have that

$$
\begin{aligned}
\|B(t) B(t-1)\|= & \max \left\{\sum_{i=1}^{2}\left|\phi_{i}+\sum_{j=1}^{2} b_{i j} Z_{t-1-j}\right|, \mid\left(\phi_{1}+\sum_{j=1}^{2} b_{1 j} Z_{t-j}\right)\left(\phi_{1}+\sum_{j=1}^{2} b_{1 j} Z_{t-1-j}\right)\right. \\
& \left.+\left(\phi_{2}+\sum_{j=1}^{2} b_{2 j} Z_{t-j}\right)|+|\left(\phi_{1}+\sum_{j=1}^{2} b_{1 j} Z_{t-j}\right)\left(\phi_{2}+\sum_{j=1}^{2} b_{2 j} Z_{t-1-j}\right) \mid\right\} .
\end{aligned}
$$

Hence, $E \ln \|B(t) B(t-1)\|<0$, if for some $\gamma>0$,

$$
\begin{gathered}
E\left\{\left[\sum_{i=1}^{2}\left|\phi_{i}+\sum_{j=1}^{2} b_{i j} Z_{t-1-j}\right|+\left|\left(\phi_{1}+\sum_{j=1}^{2} b_{1 j} Z_{t-j}\right)\left(\phi_{1}+\sum_{j=1}^{2} b_{1 j} Z_{t-1-j}\right)+\left(\phi_{2}+\sum_{j=1}^{2} b_{2 j} Z_{t-j}\right)\right|\right.\right. \\
\left.\left.+\left|\left(\phi_{1}+\sum_{j=1}^{2} b_{1 j} Z_{t-j}\right)\left(\phi_{2}+\sum_{j=1}^{2} b_{2 j} Z_{t-1-j}\right)\right|\right]^{\gamma}\right\}
\end{gathered}
$$

is less than 1 . When the noise sequence $\left\{Z_{t}\right\}$ has infinite variance but $E\left|Z_{1}\right|^{\gamma}<\infty$ for some 
$0<\gamma \leqq 1$, the following simpler condition

$$
E\left|\phi_{1}+\sum_{j=1}^{2} b_{1 j} Z_{j}\right|^{\gamma}+E\left|\phi_{2}+\sum_{j=1}^{2} b_{2 j} Z_{j}\right|^{\gamma}<1 / 2,
$$

or its slightly stronger version

$$
\sum_{i=1}^{2}\left|\phi_{i}\right|^{\gamma}+\sum_{i=1}^{2} \sum_{j=1}^{2}\left|b_{i j}\right|^{\gamma} E\left|Z_{1}\right|^{\gamma}<1 / 2,
$$

will imply the results of Theorem 1 .

The above example generates the following simpler existence condition.

Corollary 1 . If there exists a $\gamma$ with $0<\gamma \leqq 1$ such that

$$
\sum_{i=1}^{p} E\left|\phi_{i}+\sum_{j=1}^{l} b_{i j} Z_{t-j}\right|^{\gamma}<p^{-1},
$$

then condition (2) is satisfied and hence the results of Theorem 1 follow. In the case when $\left\{Z_{t}\right\}$ has finite variance $\sigma^{2}$ and zero mean, a simpler sufficient condition is

$$
p E\left\{\left[\sum_{i=1}^{p}\left|\phi_{i}+\sum_{j=1}^{l} b_{i j} Z_{j}\right|\right]^{2}\right\}<1
$$

while a slightly stronger condition is

$$
p^{2} \sum_{i=1}\left[\phi_{i}^{2}+\sum_{i j} b_{i j}^{2} \sigma^{2}\right]<1
$$

When the general bilinear model (1) reduces to the simple model, $X_{t}=Z_{t}+b_{11} X_{t-1} Z_{t-1}$, a case studied by Quinn (1982), the sufficient stationary condition, $\ln \left|b_{11}\right|+E \ln \left|Z_{t}\right|<0$ holds. Clearly, this is a special case of (2) with $p=1$ in Theorem 1.

Similarly, one can interchange the positions of $X_{t}$ and $Z_{t}$ in the above causality conditions to get the invertibility conditions for the corresponding bilinear model, provided that there already exists a strictly stationary and ergodic bilinear time series $\left\{X_{t}\right\}$ satisfying the bilinear equations involved. Hence, the duality between causality and invertibility of $\operatorname{ARMA}(p, q)$ models also carries over to the bilinear case. Interestingly, for the appropriate choice of a non-zero $b$, the model

$$
X_{t}=Z_{t}+b X_{t-1} Z_{t-1}
$$

which also has the element $Z_{t}+b Z_{t-1}^{2}$, can be invertible while the simple non-linear model

$$
Y_{t}=Z_{t}+b Z_{t-1}^{2}
$$

is non-invertible, as demonstrated by Granger and Anderson (1978b).

\section{References}

Granger, C. W. J. AND Anderson, A. (1978a) An Introduction to Bilinear Time Series Models. Vandenhoeck and Ruprecht, Göttingen.

Granger, C. W. J. AND ANDERSEN, A. (1978b) On the invertibility of time series models. Stoch. Proc. Appl. 8, 87-92.

LIU, J. (1989) A simple condition for the existence of some stationary bilinear time series. J. Time Series Anal. 10, 33-39. 
Liu, J. ANd Brockwell, P. J. (1988) On the general bilinear time series model. J. Appl. Prob. 25, 553-564.

QuinN, B. G. (1982) Stationarity and invertibility of simple bilinear models. Stoch. Proc. Appl. 12, 225-230.

Subba Rao, T. AND Gabr, M. M. (1984) An Introduction to Bispectral Analysis and Bilinear Time Series Models. Lecture Notes in Statistics 24, Springer-Verlag, New York. 